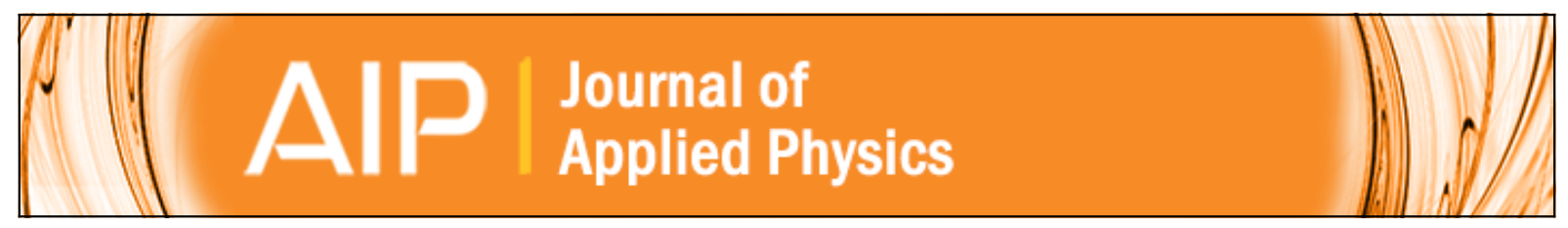

\title{
X-ray spectrometry investigation of electrical isolation in GaN
}

S. O. Kucheyev, M. Toth, M. R. Phillips, J. S. Williams, C. Jagadish, and G. Li

Citation: Journal of Applied Physics 91, 3940 (2002); doi: 10.1063/1.1452759

View online: http://dx.doi.org/10.1063/1.1452759

View Table of Contents: http://scitation.aip.org/content/aip/journal/jap/91/6?ver=pdfcov

Published by the AIP Publishing

\section{Articles you may be interested in}

Ar+-irradiation-induced damage in hydride vapor-phase epitaxy GaN films

J. Vac. Sci. Technol. A 33, 043002 (2015); 10.1116/1.4922593

Investigation of deep levels in bulk GaN material grown by halide vapor phase epitaxy

J. Appl. Phys. 114, 153702 (2013); 10.1063/1.4825052

Electrical field effect of $\mathrm{H}$-implantation induced defect states in GaN

Appl. Phys. Lett. 82, 403 (2003); 10.1063/1.1539281

Model for electrical isolation of $\mathrm{GaN}$ by light-ion bombardment

J. Appl. Phys. 92, 5740 (2002); 10.1063/1.1512690

Electrical characterization of two deep electron traps introduced in epitaxially grown n- GaN during He-ion irradiation

Appl. Phys. Lett. 73, 3745 (1998); 10.1063/1.122881

\section{AIP $\mid$ APL Photonics}

APL Photonics is pleased to announce Benjamin Eggleton as its Editor-in-Chief

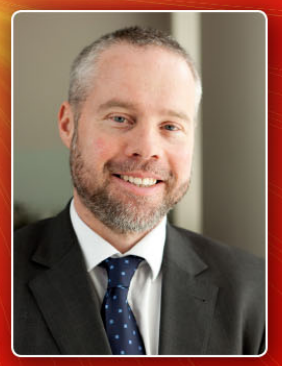




\title{
X-ray spectrometry investigation of electrical isolation in GaN
}

\author{
S. O. Kucheyeva) \\ Department of Electronic Materials Engineering, Research School of Physical Sciences and Engineering, \\ The Australian National University, Canberra, ACT 0200, Australia \\ M. Toth \\ Cavendish Laboratory, University of Cambridge, Madingley Rd., Cambridge, CB3 OHE, United Kingdom \\ M. R. Phillips \\ Microstructural Analysis Unit, University of Technology, Sydney, Broadway, NSW 2007, Australia
}

J. S. Williams and C. Jagadish

Department of Electronic Materials Engineering, Research School of Physical Sciences and Engineering, The Australian National University, Canberra, ACD 0200, Australia

G. Li

Ledex Corporation, No. 9, Ta-Yio First St., Ta-Fa Industrial District, Kaohsiung County, Taiwan, Republic of China

(Received 29 October 2001; accepted for publication 2 January 2002)

\begin{abstract}
Electrical isolation of $n$-type $\mathrm{GaN}$ epilayers bombarded with $\mathrm{MeV}$ light ions is studied by energy dispersive $\mathrm{x}$-ray spectrometry (EDS). We show that the maximum bremsstrahlung x-ray energy (the Duane-Hunt limit) can be used to monitor the isolation process in GaN. This method allows the dose region above the threshold dose for isolation to be conveniently studied, whereas the application of conventional (low-voltage) electrical techniques in this dose range with large sheet resistances of the material ( $\geqslant 10^{11} \Omega / \mathrm{sq}$ ) is often impossible due to comparable parasitic resistances of the experimental setup. A correlation of EDS and resistance measurements of GaN strongly suggests that the magnitude of sample charging scales with the number of ion-beam-produced deep electron traps which are empty at equilibrium. The results presented demonstrate the utility of EDS as a powerful and simple technique to study electrical isolation in wide band-gap semiconductors. (C) 2002 American Institute of Physics.
\end{abstract}

[DOI: $10.1063 / 1.1452759$ ]

Ion bombardment is an important technological tool for electrical isolation of compound semiconductors. ${ }^{1}$ Under specific conditions, ion irradiation can render the material highly resistive due to the formation of defect-related deep carrier traps. The isolation process can often be characterized by the so-called threshold dose $\left(D_{\mathrm{th}}\right)$, which is the ion dose necessary to produce an effective concentration of deep level traps comparable to the initial concentration of free carriers. After ion irradiation to $D_{\text {th }}$, material resistivity is close to its maximum value. This threshold dose depends on both material properties and implant conditions [such as ion energy, mass, implantation temperature, and (possibly) beam flux $]^{2}$ For effective and thermally stable electrical isolation of a particular semiconductor device structure, it is important to study the dose range close to and above $D_{\text {th }}$. Indeed, the optimum ion dose is usually above $D_{\text {th }}$ to ensure that electrical isolation is thermally stable during subsequent lowtemperature annealing.

Electrical isolation by ion irradiation has recently been applied for GaN, a technologically important wide band-gap semiconductor. ${ }^{2-7}$ In particular, it has been shown that GaN thin films can be rendered highly resistive by $\mathrm{MeV}$ light-ion irradiation ${ }^{2}$ when projected ion ranges are significantly larger than film thickness, and depth profiles of ion-beam-generated

${ }^{a)}$ Electronic mail: sergei.kucheyev@anu.edu.au atomic displacements are essentially uniform within $\mathrm{GaN}$ epilayers. However, previous studies ${ }^{2}$ have shown that isolation measurements of $\mathrm{GaN}$ for ion doses above $D_{\text {th }}$ are often difficult due to very large values of sheet resistance $\left(R_{s}\right.$ $\left.\geqslant 10^{11} \Omega / \mathrm{sq}\right)$ in this dose range. This arises for a material with a large band gap $(\sim 3.4 \mathrm{eV}$ for $\mathrm{GaN})$, and, hence, very poor intrinsic conductivity, since resistances to be measured are of the same order of magnitude as typical parasitic resistances of the experimental setup.

In this paper, we present a simple (contactless) method to study electrical isolation in $\mathrm{GaN}$ for ion doses above $D_{\text {th }}$ when the application of conventional electrical techniques is often impossible. The method is based on the fact that GaN rendered highly resistive by ion irradiation can exhibit strong charging effects during imaging in the secondary electron microscope (SEM). ${ }^{8}$ A number of different techniques have previously been applied to study (undesirable) charging effects in insulators during SEM imaging, such as the mirror method and methods based on energy shifts in Auger lines or in high-energy cutoff of the x-ray bremsstrahlung [the socalled Duane-Hunt limit (DHL)]. ${ }^{9}$ In the present study, the electrical isolation process in $\mathrm{GaN}$ is monitored by measuring the DHL, which reflects electron trapping at deep defect levels produced by ion bombardment. Hence, this study demonstrates that charging phenomena, which are often undesirable during imaging in the electron microscope, can be 


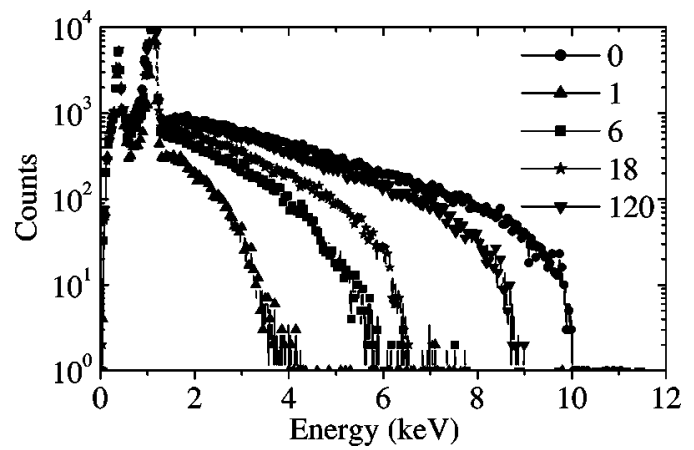

FIG. 1. EDS spectra acquired from GaN (wafer A) irradiated at room temperature with $1.8 \mathrm{MeV} \mathrm{He}$ ions with a beam flux of $\sim 6 \times 10^{12} \mathrm{~cm}^{-2} \mathrm{~s}^{-1}$ to different doses. Ion doses (in $10^{14} \mathrm{~cm}^{-2}$ ) are indicated in the legend.

used to conveniently monitor electrical isolation in wide band-gap semiconductors, such as GaN.

The $\sim 2$ - $\mu$ m-thick wurtzite $n$-type GaN epilayers used in this study were grown on $c$-lane sapphire substrates by metalorganic chemical vapor deposition (MOCVD) in a rotating disk reactor at Ledex Corporation. Wafers with roomtemperature free electron concentrations of $\sim 1 \times 10^{16}$ (nominally undoped wafers $\mathrm{A}$ and $\mathrm{B}$ ) and $\sim 3 \times 10^{17} \mathrm{~cm}^{-3}(\mathrm{Si}-$ doped wafer C) were used. Samples were irradiated at room temperature with $1.8 \mathrm{MeV}{ }^{4} \mathrm{He}^{+}$ions with a beam flux of $\sim 6 \times 10^{12} \mathrm{~cm}^{-2} \mathrm{~s}^{-1}$ using the ANU $2 \mathrm{MV}$ Van de Graaff accelerator or with $6.6 \mathrm{MeV}{ }^{12} \mathrm{C}^{+}$ions with a beam flux of $\sim 6.4 \times 10^{10} \mathrm{~cm}^{-2} \mathrm{~s}^{-1}$ using an ANU 1.7 MV tandem accelerator (NEC, 5SDH-4). Ion energies were chosen to place the damage peak deep in the sapphire substrate, beyond the GaN layer. In this case, the distribution of ion-beam-generated atomic displacements is essentially uniform throughout the GaN film. During bombardment, samples were tilted by $7^{\circ}$ off the surface normal direction to minimize channeling. Selected samples were also subjected to conventional furnace post-ion-irradiation annealing for $1 \mathrm{~h}$ at temperatures up to $700{ }^{\circ} \mathrm{C}$ in a nitrogen ambient at atmospheric pressure.

After ion irradiation, samples were studied by SEM and energy dispersive $\mathrm{x}$-ray spectrometry (EDS). Electron images and EDS spectra were obtained using a Philips XL30 environmental SEM equipped with an EDAX DX-4 EDS system. Imaging conditions (high-vacuum mode, electron energy $=10 \mathrm{keV}$, working distance $=10 \mathrm{~mm}$, scan size $=70 \times 50 \mu \mathrm{m}^{2}$, and scan rate $=10$ frames/s) were kept constant during acquisition of the EDS data shown below. The electron beam current and x-ray spectrum acquisition time (200 live seconds per spectrum) were selected so as to attain satisfactory signal-to-noise ratios in x-ray data (counting rate $\cong 2000$ c.p.s.) and were also kept constant.

It has previously been shown ${ }^{8}$ that $\mathrm{GaN}$ rendered highly resistive by $\mathrm{MeV}$ light-ion irradiation exhibits strong electric field assisted secondary electron emission (i.e., charging effects). Our present results show that the strength of such charging effects (monitored by EDS measurements) depends on implant and post-ion-irradiation conditions. Below, we first present results of EDS characterization of GaN irradiated with $\mathrm{MeV}$ light ions and, then, show a correlation of EDS data with resistance measurements.
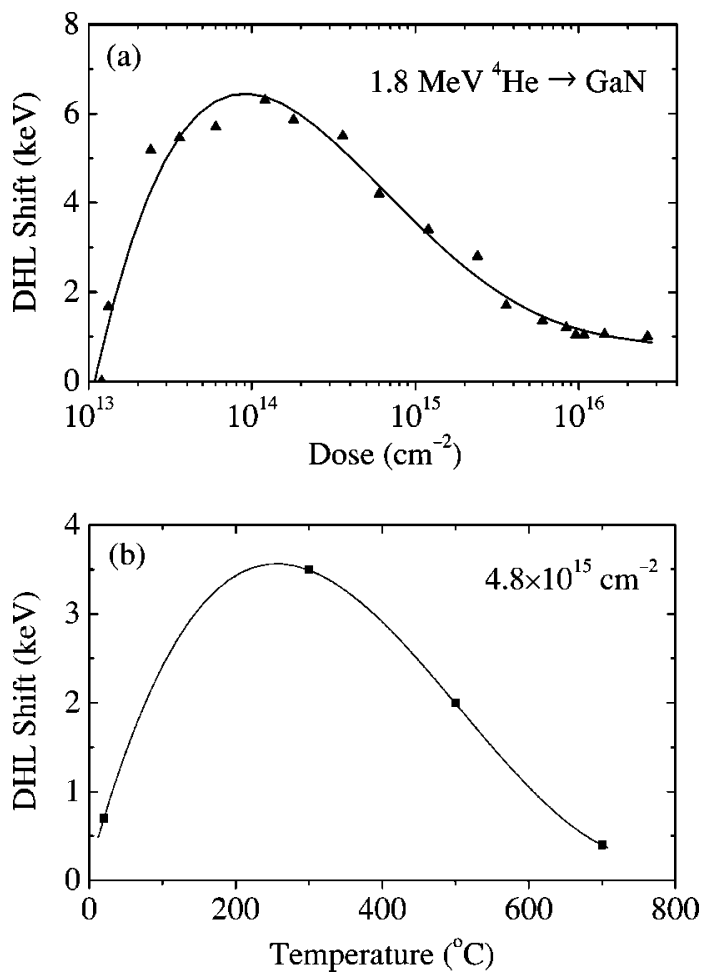

FIG. 2. (a) DHL shift as a function of ion dose for $1.8 \mathrm{MeV} \mathrm{He}$ ion bombardment of $\mathrm{GaN}$ (wafer $\mathrm{A}$ ) at room temperature with a beam flux of $\sim 6$ $\times 10^{12} \mathrm{~cm}^{-2} \mathrm{~s}^{-1}$. (b) DHL shift as a function of annealing temperature for samples (wafer B) bombarded at room temperature with $1.8 \mathrm{MeV} \mathrm{He}$ ions to a dose of $\sim 4.8 \times 10^{15} \mathrm{~cm}^{-2}$ with a beam flux of $\sim 6 \times 10^{12} \mathrm{~cm}^{-2} \mathrm{~s}^{-1}$. Postirradiation annealing was performed for $1 \mathrm{~h}$ in a nitrogen atmosphere.

Figure 1 shows typical EDS spectra acquired from GaN irradiated with $1.8 \mathrm{MeV} \mathrm{He}$ ions to different doses. It is seen from Fig. 1 that, for the as-grown sample, the DHL is $\sim 10$ $\mathrm{keV}$, the electron energy used to acquire the spectra. Conversely, spectra acquired from implanted samples clearly exhibit DHL values less than $10 \mathrm{keV}$. This decrease in the DHL results from the slowing down of the primary electrons, before they enter the sample, by an electric field above the sample. ${ }^{10}$ Such an electric field consists of two distinct components, produced during electron irradiation, as a result of (i) secondary electron emission and (ii) electrons injected and trapped in the material. ${ }^{11}$ A decrease in the DHL indicates a net negative surface potential generated by trapped electrons. $^{12,13}$

Figure 2(a) illustrates the ion dose dependence of the DHL shift (i.e., primary electron energy minus the DHL) in GaN samples irradiated at room temperature with $1.8 \mathrm{MeV}$ He ions. It is seen from Fig. 2(a) that, with increasing ion dose, the DHL shift rapidly increases after a dose of $\sim 10^{13} \mathrm{~cm}^{-2}$, reaches the maximum, and then exhibits a decrease with further ion irradiation. Shown in Fig. 2(b) is the DHL shift as a function of annealing temperature for GaN samples bombarded at room temperature with $1.8 \mathrm{MeV} \mathrm{He}$ ions to a dose of $\sim 4.8 \times 10^{15} \mathrm{~cm}^{-2}$, which is larger than the dose corresponding to the maximum of the DHL shift. Figure 2(b) reveals that, with increasing temperature of post-ionirradiation annealing, the DHL shift first increases and then exhibits a decrease. 


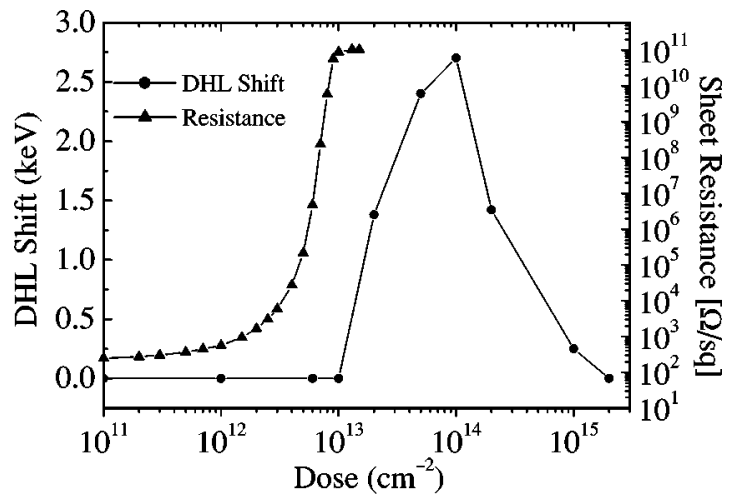

FIG. 3. Dose dependences of sheet resistance (the right axis, taken from Ref. 2) and DHL shift (the left axis) for irradiation of GaN (wafer C) at room temperature with $6.6 \mathrm{MeV} \mathrm{C}$ ions with a beam flux of $\sim 6.4$ $\times 10^{10} \mathrm{~cm}^{-2} \mathrm{~s}^{-1}$

These main features of dose and annealing temperature dependences of the DHL shift illustrated in Fig. 2 are typical for ion dose and annealing temperature curves of $R_{s}$ for a number of semiconductors. ${ }^{1}$ For example, with increasing ion dose, $R_{s}$ of $\mathrm{GaN}$ exhibits a fast nonlinear increase due to the trapping of carriers at ion-beam-produced defects together with damage-induced degradation of carrier mobility. ${ }^{2}$ With further increasing ion dose, $R_{S}$ is expected to decrease due to the onset of hopping conduction with increasing density of ion-beam-produced defects. ${ }^{1}$ In addition, for ion doses above $D_{\text {th }}$, post-ion-irradiation annealing [Fig. 2(b)] results in, first, an increase in $R_{s}$ (due to a partial annealing of ion-beam-produced defects, resulting in a reduction in hopping conduction) followed by a decrease in $R_{S}$ (due to a further annealing of deep traps responsible for electrical isolation).

The above comparison of data from Fig. 2 with (expected) ion dose and annealing temperature dependences of $R_{s}$ suggests that the DHL shift reflects the magnitude of $R_{s}$ of GaN. The correlation between $R_{s}$ and the DHL shift is quantified in Fig. 3, which shows ion dose dependences of these two parameters for $\mathrm{GaN}$ bombarded at room temperature with $6.6 \mathrm{MeV} \mathrm{C}$ ions. It is seen from Fig. 3 that a fast increase in the DHL shift occurs for ion doses above $D_{\text {th }}$ (i.e., $\geqslant 10^{13} \mathrm{~cm}^{-2}$ in this case). ${ }^{14}$ This gives a compelling argument that, for ion doses above $D_{\text {th }}$ and below the onset of pronounced hopping condition, the DHL shift scales with the number of ion-beam-produced electron traps which are empty at equilibrium. Indeed, during electrical isolation of $n$-type $\mathrm{GaN}$, all deep traps produced by ion bombardment to doses below $D_{\text {th }}$ are filled with electrons supplied by shallow donors. Figure 3 shows that the DHL shift exhibits a fast increase only for ion doses above $D_{\text {th }}$ when all free carriers supplied by shallow donors are trapped at ion-beamproduced deep levels. At such doses, the chemical potential moves toward the middle of the band gap, and some deep traps are empty (at equilibrium). ${ }^{15}$ These empty deep levels trap electrons injected into the material during SEM imaging, resulting in strong charging effects accompanied by a shift in the DHL. With further increasing ion dose, the number of deep traps which are empty at equilibrium also increases, resulting in a corresponding increase in the DHL shift. Figures 2(a) and 3 reveal a decrease in the DHL shift with further ion irradiation $\left(\geqslant 10^{14} \mathrm{~cm}^{-2}\right)$. This decrease can be attributed to hopping conduction.

The above results demonstrate that simple DHL measurements give an ideal opportunity to study electrical isolation in $\mathrm{GaN}$ for ion doses above $D_{\text {th }}$. However, it should be noted that the magnitude of DHL shifts caused by charging cannot be always directly proportional to the surface potential of the charged sample. Such nonlinearities may result from artifacts in EDS spectra, as has been discussed in Ref. 16. However, where necessary, high precision quantitative surface potential measurements can be obtained using other techniques such as secondary or Auger electron spectroscopy. ${ }^{16}$

In conclusion, we have shown that EDS measurements can be used to study the process of electrical isolation in GaN. In particular, this method allows the dose region above the threshold dose for isolation to be conveniently studied, whereas the application of conventional electrical techniques is often impossible. In addition, such contactless EDS measurements allow the whole semiconductor wafer to be studied without cutting and give an ideal opportunity to study electrical isolation in some cases when the fabrication of ohmic contacts is not feasible. Results suggest that the DHL shift reflects the number of ion-beam-produced deep electron traps which are empty at equilibrium. This simple technique may prove useful in studies of electrical isolation in other wide band-gap semiconductors.

${ }^{1}$ See, for example, a review by S. J. Pearton, Mater. Sci. Rep. 4, 313 (1990), and references therein.

${ }^{2}$ H. Boudinov, S. O. Kucheyev, J. S. Williams, C. Jagadish, and G. Li, Appl. Phys. Lett. 78, 943 (2001).

${ }^{3}$ S. C. Binari, H. B. Dietrich, G. Kelner, L. B. Rowland, K Doverspike, and D. K. Wickenden, J. Appl. Phys. 78, 3008 (1995).

${ }^{4}$ S. J. Pearton, C. B. Vartuli, J. C. Zolper, C. Yuan, and R. A. Stall, Appl. Phys. Lett. 67, 1435 (1995).

${ }^{5}$ S. J. Pearton, R. G. Wilson, J. M. Zavada, J. Han, and R. J. Shul, Appl. Phys. Lett. 73, 1877 (1998).

${ }^{6}$ C. Uzan-Saguy, J. Salzman, R. Kalish, V. Richter, U. Tish, S. Zamir, and S. Prawer, Appl. Phys. Lett. 74, 2441 (1999).

${ }^{7}$ X. A. Cao, S. J. Pearton, G. T. Dang, A. P. Zhang, F. Ren, R. G. Wilson, and J. M. Van Hove, J. Appl. Phys. 87, 1091 (2000).

${ }^{8}$ M. Toth, S. O. Kucheyev, J. S. Williams, C. Jagadish, M. R. Phillips, and G. Li, Appl. Phys. Lett. 77, 1342 (2000).

${ }^{9}$ See, for example, M. Belhaj, S. Odof, K. Msellak, and O. Jbara, J. Appl. Phys. 88, 2289 (2000), and references therein.

${ }^{10}$ L. Reimer, Scanning Electron Microscopy. Physics of Image Formation and Microanalysis (Springer, Berlin, 1985).

${ }^{11}$ J. Cazaux, J. Appl. Phys. 85, 1137 (1999).

${ }^{12}$ The polarity and magnitude of charging exhibited by a resistive sample in a SEM depend on operating parameters such as beam energy, beam current, scan speed, magnification, and sample tilt. ${ }^{13}$ In this work, these parameters were selected so as to yield a net negative surface potential (caused by charging) and were fixed for each set of measurements.

${ }^{13}$ See, for example, D. C. Joy and C. S. Joy, Micron 26, 247 (1996).

${ }^{14}$ It should be noted that measurements of GaN resistance for ion doses above $D_{\text {th }}$ are impossible due to parasitic resistances of the experimental setup. ${ }^{2}$

${ }^{15}$ In our scenario, ion-beam-induced formation of defects with shallow levels is neglected. Although this assumption may not be strictly correct, Fig. 3 indicates that the introduction rate of deep levels, trapping free carriers, is larger than the rate of creation of defects with shallow levels. Indeed, Fig. 3 shows that $R_{s}$ monotonically increases with increasing ion dose.

${ }^{16}$ M. Belhaj, Appl. Surf. Sci. 177, 58 (2001). 